\title{
Die Abbesteller des Salzburger „Rupertusblatts“
}

\author{
von Franz Rest
}

\begin{abstract}
Vorwort
Medien kämpfen um den Leser. Sie tun es mitunter mit hohem finanziellem Aufwand, mit Postwurfsendungen und inhaltlichen Schwerpunkten. Das Augenmerk wird auf die potentielle Kundschaft gelegt. Medienschaffende wissen, daß auch die Leser-Blatt-Bindung von großer Bedeutung ist und setzen diesbezügliche Aktionen. Selten hingegen konzentriert sich das Interesse auf die Abbesteller.
\end{abstract}

Das „Rupertusblatt“, die Kirchenzeitung der Erzdiözese Salzburg, wollte der Frage nachgehen, warum Menschen die Kirchenzeitung abbestellen. Bei redaktionellen Rückfragen bei etwa 300 Stornos eines Jahres stellte sich heraus, daß etwa ein Drittel davon wegen Todesfalls, hohen Alters oder schlechten Gesundheitszustandes zu verzeichnen war. Ein Drittel der Abbesteller gab keine Beweggründe für ihren Schritt an, ein Drittel fiel nach Werbeaktionen ab, wurde wegen schlechter Zahlungsmoral seitens des Verlages storniert, gab an, kein oder wenig Interesse an der Kirche, kirchlichen Themen und damit an der Kirchenzeitung zu haben, aus der Kirche ausgetreten zu sein, oder ohnedies andere katholische Publikationen im Hause zu haben. Dieser gewiß oberflächlichen Befragung stellte die „Linzer Kirchenzeitung “ eine Grafik bei, aus der eine klare Parallelität zwischen Rückgang des sonntäglichen Kirchenbesuches und dem Absinken der Kirchenzeitungs-Auflage herauszulesen war. Allerdings zeigt sich die Kurve bei den Kirchenzeitungen nicht so dramatisch wie beim Kirchenbesuch, was im übrigen auch eine Untersuchung in der BRD deutlich macht.

Das „Rupertusblatt“ wollte es genau wissen: Sind Abbesteller mit dem Produkt unzufrieden oder entspricht der Rückgang der Auflage überhaupt einem Trend in der heutigen Gesellschaft, der die Menschen eher von der Kirche weg als zu ihr hin bringt? Wir beauftragten das Institut für Publizistik an der Universität Salzburg mit der Durchführung einer Abbesteller-Befragung und erarbeiteten mit Dr. Franz Rest einen umfangreichen Fragebogen, der sowohl auf Produktkritik als auch auf Fragen der Kirchlichkeit der Abbesteller hinzielen sollte. Es wurden Adressen von Abbestellern nach Zufallskriterien ausgesucht und von Mitarbeitern des Instituts ausführliche Interviews gehalten. Das Ergebnis präsentiert im folgenden der Verantwortliche für die Befragung. Konsequenzen daraus zu ziehen, ist nun die Aufgabe der Redaktion. Auch die Konferenz der österreichischen Kirchenpresse wird sich mit dem Ergebnis auseinandersetzen. Etliche (oder die meisten) Problemfelder des "Rupertusblattes“ und seines Leserkreises finden sich auch bei den anderen Kirchenzeitungen, die ebenfalls größte Mühe an den Tag legen müssen, Abgänge durch Neuwerbungen abzufangen.

Die Abbestellerbefragung - übrigens die erste dieser Art - bildet in Zusammenhang mit diversen Leser- und Feldbefragungen eine wesentliche Hilfe zur Selbstbesinnung, zur eigenen Produktprüfung und zu allgemeinen kirchlichen Überlegungen.

Ziel und Aufgabe der Kirchenpresse ist es ja, Informations- und Kommunikationsorgan der diözesanen Kirche zu sein und einen Verkündigungsauftrag zu erfüllen. Dies - und gewiß auch die wirtschaftliche Situation - macht eine Auseinandersetzung mit Lesern und Abbestellern notwendig und verlangt auch stets Mühen, neue Leserschichten zu erreichen und weitere Menschen an den "runden Tisch der Diözese" (Communio et progressio) zu bitten. Dem wollte die Abbesteller-Befragung dienlich sein und das Ergebnis kann - falls die Konsequenzen richtig gesetzt werden - auch hilfreich sein.

Nachdem die Zeitung als solche ja öffentliche Meinung in der Kirche schafft und verbreitet, ist sie selber in der Öffentlichkeit im Gespräch. Es ist der Redaktion daher auch kein Problem, die durch die Abbesteller-Befragung erhaltenen - in erster Linie für die eigene Zeitung selbst interessanten - Antworten auch der Öffentlichkeit bekanntzugeben. So erteilte ich gerne die Zustimmung zur Publikation dieser Arbeit.

Bernhard Strobl

Chefredakteur „Rupertusblatt“ 
Die Feldarbeit für die vorliegende Befragung wurde im Zeitraum zwischen August und November des Jahres 1988 durchgeführt. Aus der Abbesteller-Kartei der Diözesanzeitung wurde eine - nach Geschlecht, Landesteil und Ortsgröße - geschichtete $\mathrm{Zu}$ fallsstichprobe gezogen. Interviews wurden in allen politischen Bezirken des Bundeslandes Salzburg und des Tiroler Anteils der Erzdiözese geführt.

Bei der Auswahl der Interviewpartner wurden von vornherein jene Abbesteller ausgeschieden, die ihr Abonnement während eines laufenden Jahres einstellten: Wir gingen davon aus, daß es sich dabei zu einem hohen Prozentsatz um Todesfälle handelt. Insgesamt wurde eine Stichprobe von 400 Personen ausgewählt, davon 45 im Tiroler Anteil der Erzdiözese.

Von den 400 ausgewählten und angeschriebenen Personen waren 178 verstorben oder verzogen. 83 Personen konnten nicht erreicht werden. 15 Personen aus der Befragungs-Stichprobe wurden entweder irrtümlich in der Abesteller-Kartei geführt, d.h. sie bezogen die Salzburger Kirchenzeitung zum Zeitpunkt der Befragung als Abonnent, oder sie hatten zwar für eine Zeit das Abonnement stillgelegt, waren inzwischen aber wieder Abonnenten der Kirchenzeitung für die Erzdiözese Salzburg geworden. Mit diesen Personen wurde daher kein Interview geführt. Mit insgesamt 123 Personen konnten intensive Befragungen durchgeführt werden.

Nicht auswertbar waren die Fragebögen aus den Gesprächen mit insgesamt fünf Personen. In die Auswertung konnten somit schließlich 118 Fragebögen berücksichtigt werden. 17 der insgesamt 118 Interviews wurden mit Kirchenzeitungs-Stornierern aus dem Tiroler Anteil der Erzdiözese durchgeführt. (Der unmittelbar an Salzburg angrenzende Teil des österreichischen Bundeslandes Tirol ist Teil der Erzdiözese Salzburg).

\section{Statistische Daten der Befragten}

An ausgewerteten Interviews standen schließlich 117 zur Verfügung. Davon waren 45 (38,5\%) mit Männern und $72(61,5 \%)$ mit Frauen geführt worden. 44,4 Prozent der Befragten waren älter als 50 Jahre.

Die Altersstruktur der befragten Abbesteller setzt sich aus folgenden Gruppen zusammen:

19 bis 29 Jahre: $8,5 \%$ der Befragten ( 10 Personen)

30 bis 39 Jahre: $21,4 \%$ der Befragten (25 Personen)

40 bis 49 Jahre: $25,6 \%$ der Befragten ( 30 Personen)

50 bis 59 Jahre: $20,5 \%$ der Befragten (24 Personen)

über 60 Jahre: $23,9 \%$ der Befragten (28 Personen)

Unter den Befragten sind die Arbeiter stark unterrepräsentiert.

Die Verteilung nach Berufsgruppen-Zugehörigkeit zeigt folgendes Bild:

26\% Hausfrauen

$20 \%$ Pensionisten/innen

18\% Angestellte/Beamte

$11 \%$ Bauern

14\% Selbstständige und Freie Berufe

8\% Arbeiter

$3 \%$ Studenten 
Die Verteilung nach der Größe des Wohnortes

Aus Gemeinden mit weniger als 2. 000 Einwohnern: $23,1 \%$

2. 000 bis 5. 000 Einwohner: $\quad 18,8 \%$

weniger als 5. 000 Einwohner:

$41,9 \%$

5. 000 bis 20. 000 Einwohner: $\quad 39,3 \%$

Aus der Stadt Salzburg: $\quad 18,8 \%$

Der formale Bildungsgrad der Befragten (höchste abgeschlossene Schule)

Volksschule

$24,8 \%$

Hauptschule

$9,4 \%$

Berufsschule

$23,9 \%$

Mittlere Schule

$19,7 \%$

Matura

$13,7 \%$

Hochschule, nicht abgeschlossen $\quad 5,1 \%$

Hochschule, abgeschlossen $\quad 3,4 \%$

\section{Das Informationsverhalten und die sozial-kulturelle Aktivität der Abbesteller}

Bei den befragten Abbestellern handelt es sich um relativ ,faule“ Printmediennutzer. Nur rund die Hälfte der Befragten (im Bundesland Salzburg 50\%, im Tiroler Anteil der Erzdiözese gar nur 42\%) sind regelmäßige - „mindestens zweimal pro Woche lesen“ Tageszeitungsleser. Die Vergleichszahlen des Bevölkerungsdurchschnittes der über 14-jährigen Personen aus der jüngsten Media-Analyse liegen bei 70 Prozent für das Bundesland Salzburg und bei 69 Prozent für Tirol.

Die Hälfte der Befragten gab auch an, nie Bücher zu lesen. Nur ein Viertel liest nach eigenen Angaben mindestens zwei oder mehr Bücher pro Jahr. Dabei gibt es keine nennenswerten Unterschiede zwischen jenen Befragten, die angaben, das Rupertusblatt vor der Stornierung des Abonnements regelmäßig gelesen zu haben (nur rund ein Drittel der befragten Abbesteller) und den deklarierten Nicht-Lesern (45\% aller Befragten) unter den Abbestellern der Salzburger Kirchenzeitung. Die regelmäßigen Rupertusblatt-Leser sind keine aktiveren Tageszeitungs- und Buch-Leser als die Nicht-Leser.

Wichtig(st)e Informationsquellen zum aktuellen politischen, sozialen und kulturellen Geschehen sind für die Mehrheit der Befragten Gespräche mit Familienmitgliedern und Freunden bzw. Bekannten. Die Abbesteller weisen eine relativ geringere aktive Teilnahme am "gesellschaftlichen " Leben, wie etwa Vereinsmitgliedschaften und -aktivitäten, auf. Teilweise ist dieses Ergebnis sicher aber auch auf den hohen Anteil an Hausfrauen und Pensionisten an den Abbestellern des Rupertusblattes zurückzuführen. Die befragten Abbesteller zeichnen sich zudem durch sehr geringe politische Aktivitäten aus.

\section{Die „Kirchlichkeit" der befragten Abbesteller}

Gottesdienstbesuch:

Rund ein Drittel der Befragten (34\%) geben an, an allen vier Sonntagen des vergangenen Monats den Gottesdienst besucht zu haben. 33 Prozent waren nach ihren Angaben allerdings an zumeist drei der vergagenen vier Sonntage beim Gottesdienst, 68 Prozent an zumindest einem.

Die Abbesteller der Kirchenzeitung weisen in der Erzdiözese Salzburg also eine Gottesdienst-Besuchs-Intensität auf, die etwa dem Durchschnitt der Salzburger Katholi- 
ken entspricht: 1987 waren es laut kirchlicher Statistik Österreichs rund 22 Prozent. Eine strenge Auslegung der Einhaltung der Sonntagspflicht ("schwere Sünde" bei „nicht jeden Sonntag in die Kirche gehen“) ist nur bei zwei Prozent der Befragten ausgeprägt. Sie stimmen mit dieser Aussage „völlig überein“. Weitere acht Prozent stimmen dieser Aussage eher zu. Im Gegensatz dazu lehnen neun von zehn der befragten Kirchenzeitungs-Abbesteller diese Position ab. (18\%: „Lehne eher ab“, 72\%: „Lehne ganz ab“). Die „Kirchlichkeit der Rupertusblatt-Stornierer ist im Durchschnitt also nicht höher als jene des Durchschnitts der Katholiken einzustufen.

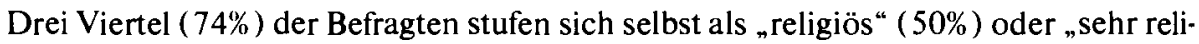
giös" (24\%) ein. Immerhin fast die Hälfte ( $45 \%$ ) der befragten Abbesteller gibt an, täglich zu beten, 19 Prozent von ihnen tun dies selten oder nie. Den Gottesdienstbesuch $(27 \%)$, oftmaligen Kommunionsempfang (22\%) und Beichte nach schwerer Sünde $(29 \%)$ hält nur etwa jeder Vierte für unbedingt notwendige Verhaltensweisen eines „guten Christen". Hier gibt es aber große Unterschiede zwischen Salzburgern und jenen Abbestellern, die im Tiroler Teil der Erzdiözese leben. Bei den Tirolern liegen die Werte bei 35 Prozent (Kirchenbesuch), 41 Prozent (oftmaliger Kommunionsempfang) und 47 Prozent (Beichte nach schwerer Sünde).

Auch das Gefälle nach der Größe des Wohnortes spricht hier eine deutliche Sprache: Personen aus Gemeinden mit weniger als 2000 Einwohnern halten diese angegebenen religiösen Aktivitäten für signifikant notwendiger für das Leben eines Christen als Personen, die in größeren Orten leben. Am wenigsten bedeutsam halten dies Abbesteller aus der Stadt Salzburg.

Hinsichtlich der Berufsgruppen, denen die Abbesteller zuzuzählen sind, gibt es einige signifikante Verschiedenheiten: Den Sonntags-Gottesdienstbesuch halten die befragten Studenten, Freiberufler und (höhere) Beamten durchwegs für eher unwichtig, wohingegen die Pensionisten, Hausfrauen und Höheren Angestellten dem Gottesdienstbesuch die relativ größte Bedeutsamkeit (im Vergleich der Berufsgruppen) für das Leben eines Christen zuzählen.

Der Großteil der befragten Abbesteller (60\%: „stimme völlig zu“, 27\%: „stimme eher $\mathrm{zu}^{\prime \prime)}$ ist der Auffassung, daß man auch ohne sonntäglichen Messebesuch ein guter Christ sein kann. 14 Prozent lehnen diese Auffassung ab. Bei Tirolern sind die Werte auch hier etwas anders: Nur 23 Prozent stimmen dieser Aussage völlig zu, und 53 Prozent „stimmen eher zu“, während diese Aussage immerhin 24 Prozent "ganz ablehnen".

Ein hoher Prozentsatz der Befragten hält den Einsatz für Arme, das „Zeit haben“ für die Mitmenschen ( $82 \%$ ), die Bereitschaft zum Verzeihen (61\%) und den „Einsatz zum Wohl anderer" (67\%) für unbedingt notwendige Eigenschaften eines Christen.

Das Interesse für Politik halten dagegen nur sieben Prozent der Befragten für eine notwendige Eigenschaft eines Christen. 62 Prozent erachten dies sogar für ausgesprochen unwichtig. Einzig die befragten Gewerbetreibenden unter den Abbestellern messen dem Interesse für Politik eine gewisse Bedeutung für das Leben eines Christen zu.

\section{Tabelle 1}

Frage: Ich lese Ihnen jetzt einige Aussagen vor, mit denen man gemeinhin einen Christen beschreiben könnte. Ich will nun nicht wissen, ob Sie diese Dinge praktizieren. Sagen Sie mir aber bitte zu jeder der folgenden Feststellungen, ob sie Ihrer Meinung nach unbedingt zum Leben eines Christen gehört, ob diese Feststellung mehr oder weniger wichtig für das Leben eines Christen ist, oder ob sie dafür eher unwichtig ist. $\mathrm{l}=$ unbedingt notwendig $2=$ mehr oder weniger wichtig $3=$ eher unwichtig bzw. unwichtig 
Er geht am Sonntag in die Kirche

Mittelwert

Er hat Zeit für seine Mitmenschen

2,068

1,171

Er verzeiht, auch wenn der andere schuldig ist

Er empfängt öfters die Kommunion

1,462

2,085

Er tritt für die Rechte der Armen und Unterdrück-

ten ein und gibt von dem, was er hat, den Armen

1,359

Seine Macht und Autorität nützt er nicht zum

eigenen Vorteil, sondern zum Wohl anderer

1,365

Er interessiert sich für Politik

2,556

Wenn er schwer sündigt, geht er beichten

2,096

\section{Aktive Mitarbeit in der Pfarre}

Nur wenige Abonnement-Stornierer zeichnen sich durch aktive Mitarbeit in der Pfarrgemeinde aus. 88 Prozent der Befragten geben an, nie bzw. nicht in der Pfarre mitzuarbeiten. Nur drei Prozent der Befragten führten an, regelmäßig in der Pfarrgemeinde mitzuarbeiten; insgesamt 12 Prozent tun dies zumindest gelegentlich. Etwa ein Drittel besucht "gelegentlich " Veranstaltungen in der Pfarre, zwei Drittel nehmen daran nicht teil. 94 Prozent kennen den Pfarrbrief ihrer Pfarre und mehr als zwei Drittel teilen mit, diesen Pfarrbrief auch regelmäßig zu lesen. 86 Prozent lesen ihn zumindest gelegentlich.

Die Pfarrgemeinderäte ihrer Pfarre sind etwa einem Drittel der Abbesteller zumindest zum Großteil bekannt. Das Kennen der Pfarrgemeinderäte ist allerdings vor allem nur in kleinen Gemeinden (unter 2000 Einwohner) zu einem hohen Prozentsatz gegeben. Die Abbesteller aus der Stadt Salzburg kennen ihre Pfarrgemeinderäte fast durchweg nicht. Hausfrauen und Bauern wissen am häufigsten, wer die Pfargemeinderäte sind. Unter den Abbestellern befanden sich zwei Pfarrgemeinderäte.

\section{Zustimmung und Ablehnung zu "Kirchlichkeits-Items"}

Tabelle 2

Frage: Welche Meinung haben Sie zu folgenden Stellungnahmen?

Antworten Sie mit: 1 = stimme völlig überein, 2 = stimme eher zu, 3 = lehne eher $a b$, 4 = lehne ganz ab

Mittelwert

Erst die Religion macht den Menschen frei und selbstbewußt

Man lebt vor allem, damit die Kinder etwas erreichen und zufrieden sind

Die Kirche soll auch in Zukunft möglichst klare Gebote und Verbote

fürdas Leben der Christen aufstellen

Die Kirche hütet ihr eigenes Vermögen, anstatt sich um Notleidende zu kümmern

Man kann auch ohne Sonntagsmesse ein guter Christ sein

Jeder soll für sich selber sorgen und niemanden mit seinen Problemen belästigen 2,788

Durch die vielen Änderungen in der Kirche wird man im Glauben unsicher

Es ist sinnlos, mit jemandem über Fragen des Glaubens zu sprechen; jeder muß für sich fühlen, was er glauben kann 
Religionsunterricht ist für die Kinder nötig,

Die viele Freiheit, die heute die jungen Leute haben, ist sicher nicht gut $\quad 2,274$

Es ist nur ein frommer Wunsch, daß Gott die Menschen liebt $\quad 3,186$

Je mehr man weiß, und je selbstständiger man wird, umso weniger

braucht man einen Glauben

Wenn man kirchlich heiratet, fühlt man sich mehr aneinander gebunden $\quad 2,122$

Wenn ein Christ nicht jeden Sonntag zur Kirche geht,

begeht er eine schwere Sünde

Die Predigt ist meistens lebensfern, seine eigenen wichtigen Probleme muß man selbst lösen

Der Sinn des Lebens besteht darin, eine angesehene Position zu gewinnen $\quad 3,088$ Im Wirtschaftsleben ist es nicht möglich, bei der Wahrheit zu bleiben

41 Prozent der Befragten wünschen sich klare Gebote und Verbote der Kirche, weitere 27 Prozent stimmen diesem Wunsch "eher zu“. Daneben meint ein Drittel der Befragten ( $29 \%$,stimme völlig zu“, $9 \%$,stimme eher $z u^{\prime}$ ), daß jeder ,selber fühle, was er glauben kann“. Der Wunsch nach klaren Geboten und Verboten der Kirche nimmt mit der Zunahme der Einwohnerzahl des Wohnorts signifikant ab.

Dem Satz, „der Kirche muß man mit Ehrfurcht begegnen und nicht mit Kritik“, stimmt ein knappes Drittel der Befragten zu ( $19 \%$,stimme völlig überein“, $11 \%$,stimme eher

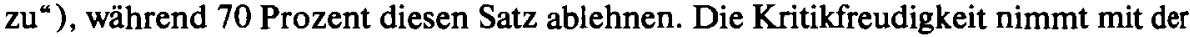
Wohnortgröße und der formalen Bildung zu; am höchsten ist sie bei den Befragten mit Matura; sie ist im Bundesland Salzburg größer als im Tiroler Anteil bei jungen höher als bei älteren. Studenten, Freiberufler und höhere Beamte lehnen den Satz am stärksten ab, Pensionisten und Gewerbetreibende stimmen ihm am stärksten zu.

Ähnliche Ergebnisse zeitigt auch die Behauptung „Gott spricht durch die Kirche ständig zu den Menschen". 69 Prozent der Befragten stimmen diesem Satz zu, ein knappes Drittel lehnt ihn ab. Auch hier steigt die Ablehnung mit der Einwohnergröße und dem formalen ( Schul-)Bildungsgrad.

Auch die Beurteilung der Notwendigkeit des Religionsunterrichts, um zu lernen "was sich gehört", ist in Orten mit geringerer Einwohnerzahl, bei Leuten mit niedrigerer formaler Bildung und mit zunehmendem Alter höher. Insgesamt wird diesem Satz von 86 Prozent der Befragten zugestimmt.

Zu große Freiheiten für die Jugend wollen 55 Prozent der Befragten erkennen. Menschen mit "nur" Volksschulbildung, Pensionisten, Einwohner von kleinen Orten und ältere stimmen dem Satz stärker zu.

Für ein Drittel der Befragten stellt die Kirche nach ihren Angaben keine Hilfe dar. Ein Fünftel der Befragten (22\%) tendiert zu der Ansicht, „daß keinen Glauben braucht, wer weiß“. Ein Viertel stimmt dem Satz, „Erfolg ist der Sinn des Lebens“ zu, drei Viertel lehnen ihn (eher) ab.

Der Moralität im Wirtschaftsleben traut weniger als die Hälfte der Befragten. Dem Satz „Im Wirtschaftsleben braucht man die Lüge“ stimmen 45 Prozent zu. 


\section{Leseintensität des Rupertusblatts - Kirchlichkeitsgrad der Abbesteller}

Die regelmäßigen Rupertusblatt-Leser unter den Abbestellern sind nicht in einem feststellbar höherem Maße als der Durchschnitt der Abbesteller auch regelmäßige Tageszeitungs- und Buchleser. Jedenfalls läßt sich aus der vorliegenden Untersuchung der Schluß nicht ziehen. Regelmäßige Rupertusblatt-Leser zeichnen sich durch eine signifikant positivere Einstellung zur Kirche und eine höhere Religiosität aus als die nur ,gelegentlichen“ Rupertusblatt-Leser und die Nichtleser. Die regelmäßigen Rupertusblatt-Leser unter den Abbestellern sind vergleichsweise fleißigere Gottesdienst-Besucher. Mehr als die Hälfte der regelmäßigen Leser gehen jeden Sonntag zum Gottesdienst, alle übrigen zumindest gelegentlich. Die Nicht-Leser unter den Abbestellern dagegen sind nur zu einem Fünftel Nicht-Gottesdienst-Besucher. Den regelmäßigen Sonntags-Gottesdienstbesuch halten die Vielleser zu 35 Prozent für "unbedingt notwendig im Leben eines Christen“ ( $12 \%$ für „(eher) unwichtig“), während die NichtLeser nur zu 19 Prozent von der Notwendigkeit des regelmäßigen Sonntagsgottesdienstbesuchs für Christen überzeugt sind. Mehr als die Hälfte der "Nicht-Leser" hält den regelmäßigen Gottesdienstbesuch sogar für „(eher) unwichtig “ für das christliche Leben. Jene Kirchenzeitugs-Abbesteller, die das Rupertusblatt regelmäßig gelesen haben, stufen sich fast zu 100 Prozent als "religiös" oder "sehr religiös" ein, während weniger als zwei Drittel der deklarierten Nicht-Leser sich als religiös beurteilen.

Die regelmäßigen Rupertusblatt-Leser weisen in allen entsprechenden Items einen bedeutend höheren Grad an Kirchlichkeit auf als jene Abbesteller, die angaben, das Rupertusblatt nur "gelegentlich“ oder "kaum/nie" gelesen zu haben. So beurteilen etwa die intensiven Rupertusblatt-Leser den Kommunionempfang zu 35 Prozent als „unbedingt notwendig" für einen Christen (nur $18 \%$ als "eher unwichtig"), wohingegen die Nicht-Leser zu 11 Prozent von der unbedingten Notwendigkeit des Kommunionempfangs für das Leben eines Christen überzeugt sind. Auch die Notwendigkeit des Beichtens nach schwerer Sünde sehen die regelmäßigen Rupertusblatt-Leser stärker gegeben (28\% „unbedingt notwendig“) als die Nicht-Leser (19\% „unbedingt notwendig“).

\section{Das Interesse an den Themen der Rupertusblatt-Berichterstattung}

Folgende Ressorts (Themenbereiche) des Rupertusblatts interessierten die Rupertusblatt-Abbesteller am stärksten:

"Information aus der Pfarre (55\% "sehr interessant“, 15\% „nicht interessant")

"Ehe und Familie (46\% „sehr interessant", 32\% „nicht interessant“)

* Praktische Lebenshilfe (46\% „sehr interessant")

* Jugendfragen (44\% "sehr interessant")

* Altersfragen (44\% „sehr interessant“)

Relativ schwaches Interesse besteht an den Themenbereichen:

* Besinnung und Meditation (57\% „nicht interessant", 19\% ,besonders interessant ${ }^{\text {*) }}$

* Wirtschaftsfragen ( $52 \%$ „nicht interessant ${ }^{*}, 16 \%$ „sehr interessant")

* Buchbesprechungen (47\% „nicht interessant", $18 \%$ „besonders interessant")

" Beiträge für Kinder ( $38 \%$,nicht interessant ", aber 35\% besonders interessant")

* Personalien und Veranstaltungen (37\% "nicht interessant", aber 36\% „besonders interessant ${ }^{\prime \prime}$ )

* Fragen der Moral (33\% „nicht interessant", 25\% „besonders interessant“)

" Kulturelles (33\% "nicht interessant", 28\% "besonders interessant")

Einem Drittel der Interviewten war das Rupertusblatt seit Kindheit an bekannt, die Hälfte von ihnen hat es erst im Erwachsenenalter kennengelernt. Ein einziger Abbe- 
steller gab an, daß er jetzt, seit er das Rupertusblatt nicht mehr abonniert hat, etwas vermisse. Nur eine einzige Befragte hat deshalb inzwischen das Rupertusblatt wieder abonniert. (Diese ist nicht im ausgewerteten Sample).

\section{Interesse an Themen der Kirchenzeitungs-Berichterstattung - Kirchlichkeit der Abonnenten-Stornierer}

Die kirchennahen Rupertusblatt-Abbesteller zeigen an den meisten Themenbereichen der Rupertusblatt-Berichterstattung ein signifikant höheres Interesse als die als kirchenfern einstufbaren Stornierer. Entsprechend nimmt das Interesse an den Rupertusblatt-Themen bei den Abbestellern mit der zunehmenden Kirchenferne ab. Eine Ausnahme stellen die Themen „Kulturelles, Mission, Dritte Welt“ und „Aktuelle Wirtschaftsfragen" dar. Hier verhält es sich umgekehrt: Die Kirchenfernen bekunden an diesen Themen signifikant höheres Interesse als die kirchennäheren Abbesteller. Das Interesse an den Themenbereichen "Leserbriefe“, "Personalnachrichten/Veranstaltungen“, "Jugendfragen“ und "Altersprobleme " weist keine signifikanten Unterschiede zwischen den kirchennäheren und kirchenferneren Rupertusblatt-Stornierern auf.

\section{Das Rupertusblatt im Urteil der Abbesteller}

Tabelle 3: Beurteilung des Rupertusblatts durch die Abbesteller

Wenn Sie an das Rupertusblatt denken, welche der folgenden Feststellungen trifft zu? Bitte sagen Sie zu jeder Aussage, ob diese sehr, kaum oder nicht auf das Rupertusblatt zutriff. 1 = trifft sehr zu; 2 = trifft einigermaßen zu; $3=$ triff kaum zu; $4=$ trifft nicht $\mathrm{zu}$ (Angaben in Prozent)

Das Rupertusblatt (RB) berichtet ausführlich aus der gesamten Kirche Mittelwert

Das RB gibt mir wertvolle Anregungen zur Kindererziehung

Das RB behandelt zu wenig die Probleme jüngerer Menschen

Das RB spricht nur strenggläubige Katholiken an

Das RB bringt zu viele Beiträge zur Dritten Welt

2,43

2,34

Das RB gibt mir Hilfen zu Besinnung und Gebet

2,92

Das RB tritt zu wenig für Sitte und Ordnung ein

2,68

Das RB sollte mehr Beiträge für Frauen bringen

3,47

Das RB sollememr $\quad 2,77$

Das RB solltemehr theologisch-religiöse Beiträge bringen

2,77

Das RB sollt weltoffener berichten

3,15

Das RB ist übersichtlich gegliedert

2,37

Die Schrift des RB ist gut lesbar

1,54

1,18

Das RB faßt zu viele heiße Eisen an

3,62

Das RB ist der Kirche gegenüber zu kritisch eingestellt

Das RB bringt zu viele Informationen und "Weihrauch" aus der Kirchenführung 3,33

Das RB bringt zuwenig Informationen aus meiner Gemeinde (und anderen Gemeinden)

Dem Rupertusblatt wird von den Abbestellern ein gutes Zeugnis ausgestellt, was die

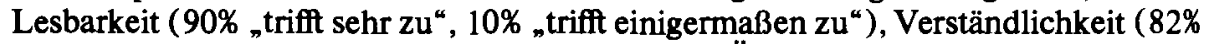
"trifft sehr $\mathrm{zu}^{\text {“ }}, 10 \%$ "trifft einigermaßen zu“) und Übersichtlichkeit (68\% „trifft sehr $\mathrm{zu}^{\text {“ }}, 24 \%$,trift einigermaßen zu“) anbelangt. Und dies, obwohl (oder weil?) immerhin $45 \%$ der Abbesteller angegeben hatten, daß sie das Rupertusblatt schon seit einiger Zeit kaum oder nie gelesen haben. 
Mehr als die Hälfte der Abbesteller (47\% „nicht“ ${ }^{4}$ 4\% „kaum“) merkten an, durch das Rupertusblatt keine Hilfe zu Besinnung und Gebet erlebt zu haben. Ein Drittel wünscht sich weniger Berichterstattung über die Dritte Welt, 38 Prozent mehr über Frauen, 28 Prozent mehr Theologisch-Religiöses. Fast zwei Drittel der Abbesteller hätten sich aber mehr "Weltoffenheit" in der Rupertusblatt-Berichterstattung gewünscht. Nur 8 Prozent meinen, das Rupertusblatt fasse zu viele "heiße Eisen" an und für nur neun Prozent trifft es „einigermaßen“ $\mathrm{zu}(0 \%$,trift sehr zu“), daß das Rupertusblatt zu kritisch zur Kirche eingestellt sei. Ebenso meinten nur 13 Prozent, daß es „einigermaßen“ zutreffe, daß durch das Rupertusblatt „zu viel Weihrauch“ über die Kirchenführung verbreitet werde.

Ein knappes Drittel der befragten Abbesteller schließlich fand im Rupertusblatt zu wenig über die Heimatgemeinde und -pfarre.

\section{Die Beurteilung der Inhalte des Rupertusblatts und die Leseintensität}

An den meisten im Rupertusblatt dargebotenen Inhaltsbereichen zeigten die regelmäBigen Rupertusblatt-Leser signifikant höheres Interesse als die "Nicht-Leser". Bei den Themenbereichen „Personalnachrichten, Veranstaltungen“, „Buchbesprechungen“, "Leserbriefe“, „Berichte über die Weltkirche“, „Erziehung(spolitik)“, „Fernsehprogramm " sind keine nennenswerten Interessensunterschiede feststellbar. An den - eher den nicht-religiösen und nicht-kirchlichen Fragen gewidmeten - Themenbereichen „Kulturelles“, „Aktuelle Wirtschaftsfragen“ sowie am Themenbereich „Mission, Dritte Welt" zeigten sich die "Nicht"- bzw. "Fast-Nie“-Leser des Rupertusblattes merkbar stärker interessiert als die regelmäßigen Rupertusblatt-Leser.

Die Untersuchung kann ferner bestätigen, daß die kirchennäheren Abbesteller merkbar höheres Interesse an jenen Themenbereichen im Rupertusblatt zeigen, die sich mit Fragen der Kirche und Religion beschättigen, als die kirchenfernere Gruppe der Abbesteller. Die Rupertusblatt-Leser zeigen auch in der Beurteilung des Rupertusblatts eine positivere Bewertung als die Nicht-Leser.

\section{Die Beurteilung des Rupertusblatts und die Kirchlichkeit der Stornierer}

Keine sehr auffallenden Unterschiede zwischen „kirchlichen“ und „säkularisierten“ Abbestellern unter den Befragten ergibt die Untersuchung hinsichtlich der Beurteilung des Rupertusblatts. Es sind nur einige klare - ohnehin "logische" - Korrelationen feststellbar:

Je größer die Kirchlichkeit der Abbesteller, desto weniger meinen sie, das Rupertusblatt sei „nur für Strenggläubige “. Der Kirchlichkeitsgrad korreliert auch mit der Beurteilung des Rupertusblatts als "Hilfe zu Besinnung und Gebet“. Den kirchenfernen Abbestellern erscheint das Rupertusblatt etwa - allerdings nur geringfïgig - "unkritischer" gegenüber der Kirche als den kirchennäheren. Und die stärker „Säkularisierten“ lesen im Rupertusblatt mehr „Weihrauch“ gegenüber der Kirchenführung, während die Kirchennahen dies praktisch nicht sehen.

Weitere stichhaltige Aussagen sind zu diesem Themenbereich allerdings nicht möglich.

\section{Die Gründe für die Stornierung des Rupertusblatt-Abonnements}

Bei der Angabe der Abbestellgründe waren Mehrfachnennungen (bis zu drei Gründe) möglich. Die Gründe wurden auch nach ihrer Wichtigkeit gereiht. Mehr als die Hälfte 
der Befragten (53\%) gab Zeitmangel als einen Abbestellgrund an, 28 Prozent der Befragten nannten diesen Grund als den wichtigsten Abbestellgrund, jeweils weitere 17 Prozent nannten ihn als zweiten oder dritten Grund der Kündigung des RupertusblattAbos.

Auf die gesondert gestellte Frage nach der persönlichen Leseintensität des Rupertusblatts hatten immerhin 45 Prozent angegeben, das Rupertusblatt als Abonnent nur selten oder nie gelesen zu haben. Nur ein knappes Drittel der befragten Abbesteller hatte nach ihren Angaben das Rupertusblatt regelmäßig gelesen.

Man kann also davon ausgehen, daß fast die Hälfte der Abbesteller den „Bruch“ mit ih. rer Kirchenzeitung bereits einige Zeit vor der Kündigung vollzogen hatte. Aber auch von den regelmäßigen Rupertusblatt-Lesern unter den Abbestellern wird Zeitmangel relativ häufig (33\% der „Intensivleser“, knapp mehr als die Hälfte der „Nie“ - oder "Fast nie“-Leser) als Abbestellgrund angegeben.

Zeitmangel wird v.a. von den 30- bis 49-jährigen als Abbestellgrund angeführt. Aber auch die über 60-jährigen ( $50 \%$ der Befragten aus dieser Altersgruppe), die Hausfrauen (48\%) und die Pensionisten (33\% von ihnen) nennen diesen Stornogrund. Am häufigsten wird diese Kündigungsursache von den Bauern angeführt.

Rund 15 Prozent der Befragten geben als Abbestellgrund die zu hohen Kosten des Rupertusblatts an, wobei für 10 Prozent der Befragten dieser Grund wichtigster Abbestellgrund war. Die Kosten sind v.a. für die älteren (über 60 Jahre) Rupertusblatt-Abbesteller wichtiger Abbestellgrund. Neben den Pensionisten (33\% der befragten Pensionisten) und Hausfrauen (20\% der befragten Hausfrauen) nennen diesen Abbestellgrund nur zwei Bauern und zwei niedrige bzw. mittlere Angestellte. Die Gruppe jener Rupertusblatt-Abbesteller, die Kostengründe für ihr Storno angaben, gehören mehrheitlich dem „kirchennahen“ Segment unter den Abbestellern an. Zwei Drittel von ihnen gibt an, jeden Sonntag den Gottesdienst zu besuchen. Sie stufen sich zu 90 Prozent als "religiös" (45\%) oder "sehr religiös" $(45 \%)$ ein.

Für Frauen hat die Kostenfrage eine signifikant höhere Bedeutung als für Männer. Kein Abbestellgrund sind die Kosten auch für die Befragten mit höherer Schulbildug (keine Nennung ab Matura-Niveau). Zwei Drittel jener Befragten, die Kostengründe angaben, weisen "nur“ Volksschulbildung auf. Von den Stadt-Salzburgern wurden Kostengründe nie angegeben. Neben der Frage nach den tatsächlich ausschlaggebenden Stornogründen wurden auch jene nach den Vermutungen über mögliche Abbestellgründe gestellt. Die Kosten des Abonnements wurden dabei von jenen Abbestellern, die eine größere Kirchlichkeit aufweisen, zu einem signifikant geringerem Maße als StornoGrund vermutet als von den Kirchenfernen. Diese Vermutung widerspricht aber den angegebenen tatsächlichen Abbestellgründen. Denn die aus Kostengründen Abbestellenden gehören fast durchwegs dem kirchennäheren Segment der befragten Stornierer an.

Fast ein Viertel (23\%) der Befragten gibt als einen Abbestellgrund an, der Pfarrbrief genüge zur Information. Diese Antwort wurde allerdings nur in zwei Fällen als wichtigster bzw. erster Abbestellgrund angegeben. Jene, die diesen Grund angaben, waren auch nur zu einem geringen Prozentsatz ( $15 \%$ von ihnen) regelmäßige RupertusblattLeser. Relativ häufiger waren es Pensionisten ( $40 \%$ der befragten Pensionisten) bzw. über 60 -jährige ( $44 \%$ von diesen).

Die Pfarrbriefe werden auch von den Kirchenzeitungs-Abbestellern zu einem sehr hohen Prozentsatz gelesen. 83 Prozent der Befragten gaben an, ihren Pfarrbrief regelmä- 
Big zu bekommen, rund zwei Drittel (64\%) lesen ihn auch regelmäßig, weiter 19 Prozent lesen ihn gelegentlich und nur 17 Prozent sind erklärte Nichtleser des Pfarrbriefes.

Ebenfalls meist nicht als Haupt-A bbestellgrund (dies nur von 7\% der Befragten) wurde genannt, das Rupertusblatt nur aus „Familientradition“ (weiter-)abonniert zu haben und nun einen längst „fälligen“ Schritt getan zu haben. Rund ein Fünftel der Antworten $(20,5 \%)$ weist hier eine Nennung auf. Diese Traditionsleser waren zu zwei Drittel allerdings bereits als Abonnenten eigentlich „Nicht" zent „Intensivleser" (=regelmäßige Rupertusblatt-Leser). Diese „Nur aus TraditionLeser $^{\star}$ und „Nun-Abbesteller ${ }^{\star}$ kommen stärker (zu zwei Drittel) aus größeren Gemeinden (über 5.000 Einwohner) bzw. aus der Stadt Salzburg (ein Drittel). Sie gehören v.a. der mittleren Altersgruppe (30- bis 50-jährige) an und haben als Ausbildung die Matura (zwei Drittel der befragten Maturanten gaben diesen Grund an; $40 \%$ aller Befragten, die diesen Grund nannten, weisen Maturaniveau auf). Sie sind keine regelmäßigen Sonntags-Gottesdienst-Besucher (nur 2 von insgesamt 24 Personen, die diesen Grund angaben, besuchen regelmäßig den Sonntags-Gottesdienst) und sie stufen sich auch zu ca. 40 Prozent als ,religiös indifferent“ ein.

12 Prozent der Befragten gaben als einen Abbestellgrund auch an, das Rupertusblatt nur "Verwandten zuliebe" abonniert zu haben. Auch die Personen aus dieser Gruppe waren mehrheitlich (mehr als zwei Drittel von ihnen) „Nicht" - oder "Fast nie "-Leser.

Grundsätzliches Desinteresse an der Kirche bzw. an kirchlichen Zeitungen gaben 14 bzw. 10 Prozent der Befragten als Abbestellgrund an. (Überwiegend Mehrfachnennung der identen Gruppe). Es sind dies mehrheitlich Männer, und sie kommen zur Hälfte aus der Stadt Salzburg. Unter ihnen sind - natürlich - keine regelmäßigen Gottesdienst-Besucher zu finden, und sie stufen sich mehrheitlich (zu 85\%) als „religiös indifferent“ bzw. „eher nicht religiös“ ein. Unter ihnen ist kein Bauer, keine Hausfrau und kein Pensionist. Sie haben zur Hälfte mindestens Maturaniveau und zu ihnen zählt kein einziger Befragter aus dem Tiroler Anteil der Erzdiözese.

Unter anderem auch nur „Verwandten zuliebe“ hatten 12 Prozent der Abbesteller das Rupertusblatt abonniert. Diese Gruppe weist hohe Übereinstimmung auf - und ist teilweise ident (Mehrfachnennungen) - mit jenen, die angaben, nur aus "Tradition" zu den Rupertusblatt-Beziehern gehört zu haben. Sie waren großteils „Nie ${ }^{\star}$ oder "Fast nie “-Leser des Kirchenblattes. Die Gruppe jener, die das Rupertusblatt unter anderem auch aus Ärger über den Pfarrer abbestellt haben (14\%) waren zu zwei Drittel „Nichtleser", weisen sonst jedoch keine interpretierbaren Abweichungen vom Durchschnitt auf.

Daß das Rupertusblatt zuwenig über den Tiroler Anteil der Erzdiözese berichte, beklagen naturgemäß nur die befragten Abbesteller aus diesem Teil der Diözese. Wenngleich die Anzahl der befragten Tiroler keine statistisch repräsentativen Aussagen zuläßt, so fällt doch auf, daß fast die Hälfte der befragten Tiroler dieses von ihnen empfundene Informationsdefizit als einen Abbestellgrund angeben. Er wird allerdings nie als erster Abbestellgrund genannt. Vier aus dieser Gruppe sind regelmäßige Gottesdienstbesucher, die restlichen vier besuchen den Gottesdienst nur an Festtagen oder nie.

Es bleibt auch anzumerken, daß die letztgenannten vier Personen den Mangel an Information über den Tiroler Anteil beklagten, obwohl sie angegeben hatten, das Rupertusblatt nur kaum oder nie gelesen zu haben.

Nur ein sehr geringer Anteil der Befragten nennt konkrete Kritik an der Kirchenzeitung als Abbestellgrund. Dazu kommt, daß diese Gründe nur in Einzelfällen als „HauptAbbestellgrund" angeführt wurden. 
Hier eine kurze Auflistung der zu diesem Bereich genannten Gründe: (Nennungen nach Frequenzreihen)

$\begin{array}{ll}\text { 1. Rupertusblatt ist nicht aktuell } & 6 \text { Nennungen } \\ \text { 2. Langweilige Aufmachung } & 6 \\ \text { 3. Rupertusblatt ist nicht objektiv } & 4 \\ \text { 4. Rupertusblatt ist zu oberflächlich } & 4 \\ \text { 5. Zu wenig informativ } & 4 \\ \text { 6. Inhalte entsprechen nicht } & 2 \\ \text { 7. Zu viele Werbeanzeigen } & 2 \\ \text { 8. Zuviel über 3. Welt } & 2 \\ \text { 9. Zu wenig über 3. Welt } & 1\end{array}$

\section{Abbestellgründe und Intensität des Rupertusblatt-Lesens}

Bei den „regelmäßigen" Rupertusblatt-Lesern unter den befragten Abbestellern wird von etwas mehr als einem Drittel der Zeitmangel als Abbestellgrund angegeben. Aber bereits der am zweithäufigsten genannte Stornogrund waren für die regelmäßigen Leser die zu hohen Kosten des Rupertusblatt-Abonnements: Jeder vierte befragte (24\%) Rupertusblatt-Abbonement-Besteller, der die Kirchenzeitung regelmäßig gelesen hatte, gibt die Kosten als einen Abbestellgrund an (im Gegensatz dazu nur 15\% aller Abbesteller).

Weitere öfter genannten Stornogründe sind für die regelmäßigen Leser das Fehlen der Ortsnachrichten (für ein Drittel dieser Gruppe; im Gegensatz dazu für 11 Prozent aller Stornierer) und die bestehende Möglichkeit, das Rupertusblatt anderswo zu lesen (22\% im Vergleich zu 7\% aller Abbesteller ein Stornogrund). Jene, die das Rupertusblatt aus „Ärger über den Pfarrer" abbestellt haben, waren mit einer einzigen Ausnahme bereits vor diesem Schritt Nicht-Leser der diözesanen Kirchenzeitung. Dieser „Ärger" dürfte daher meist ein nicht unwillkommener Anlaß zur Stornierung gewesen sein.

Nicht-Leser haben das Rupertusblatt zu einem verhältnismäßig hohen Prozentsatz nur aus Tradition oder "den Verwandten zuliebe" abonniert. Diese Abonnentengruppe hat sich - wie schon an anderer Stelle erwähnt - zu einem hohen Maße bereits früher vom Rupertusblatt verabschiedet. Die "Nur aus Tradition-Abonnierer" oder "den Verwandten zuliebe-Abonnierer" weisen auch eine wesentlich reduzierte Kirchlichkeit auf. Sie sind zu einem sehr hohen Prozentsatz keine oder nur seltene Gottesdienstbesucher. Die "Storno-Gründe der ,gelegentlichen Rupertusblatt-Leser" sind nicht als signifikant vom Durchschnitt abweichend interpretierbar.

\section{Überblick: Die wichtigsten Abbestellergruppen}

Abbestellgrund: „Das Rupertusblatt ist zu teuer“

Die Hälfte der Personen, die diesen Grund als Abbestellgrund angaben - insgesamt 15 Prozent der Befragten - bezeichneten sich als regelmäßige Rupertusblattleser, jeder füntte von ihnen gab an, das Rupertusblatt als Abonnent kaum oder nie gelesen zu haben.

Jene befragten Personen, für welche die Kosten der Kirchenzeitung einen Abbestellgrund darstellten, weisen eine relativ hohe Kirchlichkeit und Religiosität auf. Sie sind zu zwei Drittel regelmäßige Gottesdienstbesucher, meinen aber andererseits ebenfals zu zwei Drittel, daß man auch ohne regelmäßigen Gottesdienstbesuch ein "guter Christ" sein könne. Sie stimmen zu 90 Prozent dem Satz "Religion macht den Menschen frei“ ${ }^{\star} \mathrm{zu}$ und wollen zu 100 Prozent klare Gebote der Kirche. 
90 Prozent jener, welche die zu hohen Kosten des Rupertusblatts als Abbestellgrund angaben, stimmen auch mit der Auffassung überein, „Gott spricht durch die Kirche ständig zu den Menschen“. Sie halten ferner den Religionsunterricht zu 100 Prozent für wichtig und meinen ebenfalls ohne Ausnahme, daß „die viele Freiheit für die jungen Menschen nicht gut" sei. Die Kirche ist für eine große Mehrheit von ihnen eine Hilfe.

\section{Abbestellgrund: Zeitmangel}

Jene Abbesteller, die Zeitmangel anführten (53\% der Befragten), waren nur zu etwa einem Fünftel regelmäßige Leser des Salzburger Diözesanblattes. Fast die Hälfte von innen hatte das Rupertusblatt auch als Abonnent kaum oder nie gelesen. Den Gottesdienst besuchen 20 Prozent aus dieser Gruppe regelmäßig, zwei Drittel kaum oder nie. Diese Gruppe weist auch sonst eine stärkere Kirchenferne auf als der Durchschnitt der befragten Abbesteller.

Mehr als drei Viertel der Befragten aus dieser Gruppe meinen, man könne auch ohne sonntäglichen Gottesdienst ein guter Christ sein, und die Hälfte dieser Befragten stimmt dem Satz, „die Kirche vernachlässigt die Notleidenden“, zu. Rund 60 Prozent der Befragten, die diesen Abbestellgrund angaben, lehnen auch ab, daß man der Kirche "mit Ehrfurcht und nicht mit Kritik“ begegnen solle.

\section{Abbestellgrund: Informationsflut}

Hier sind wenige Aussagen über signifikante Abweichungen möglich. Nur jeder fünfte der Befragten, die diesen Stornogrund angaben, war regelmäßiger Leser. Diese Gruppe besteht je zur Hälfte aus regelmäßigen Gottesdienstbesuchern und „Nicht-Kirchengehern “ und weist auch bei anderen Kirchlichkeitskriterien eine große Polarität innerhalb der Gruppe auf: Die eine Hälfte von ihnen kann als eher kirchennah, die zweite Hälfte aber als kirchenfern angesehen werden.

\section{Abbestellgrund: Kein Interesse an der Kirche}

Die an der Kirche deklariert nicht interessierten Abbesteller waren überwiegend ( 14 von 16 Befragten) Nicht-Leser. Sie weisen durchgehend klare Merkmale der Kirchenferne auf: 14 von 16 Befragten, die diesen Grund nannten, gaben auch an, nie den Sonntags-Gottesdienst zu besuchen. Sie lehnen zum gleichen Prozentsatz die Auffassung ab, die "Religion macht den Menschen frei ${ }^{\star}$. Ebenfalls 14 der 16 Befragten aus dieser Gruppe lehnen klare Gebote der Kirche ab; keiner von ihnen meint allerdings, die Kirche vernachlässige die Notleidenden. Die erwähnten - vermutlich weitgehend selben 14 Befragten aus dieser Gruppe lehnen auch die Auffassung ab, der Kirche müsse man mit Ehrfurcht und nicht mit Kritik begegnen. Alle Befragten aus dieser AbbestellerGruppe lehnen auch den Satz „Gott spricht durch die Kirche ständig zu den Men$\operatorname{schen}^{\prime} \mathrm{ab}$.

\section{Abbestellgrund: Kein Interesse an der Religion}

Auch die an der Religion Uninteresssierten (dies gaben allerdings nur vier Personen als Abbestellgrund an) sind durchwegs Nicht-Leser und Nicht-Gottesdienst-Besucher, die (aufgrund der Mehrfachnennungen) offensichtlich ein Teilsegment bzw. den "harten Kern" aus der obengenannten Gruppe darstellen. Sie halten nichts von Ehrfurcht vor der Kirche und meinen, daß jeder selbst fühle, was er glauben soll. Folgerichtig lehnen sie auch durchweg ab, daß ,Gott durch die Kirche spricht ${ }^{\text {“; }}$ die Kirche ist diesen Menschen durchweg keine Hilfe. 
Abbestellgrund: Kein Interesse an kirchlichen Zeitungen

Auch diese Gruppe der Abbesteller korreliert stark mit den beiden vorhergenannten Gruppen. Alle zwölf Befragten, die diesen Grund als Abbestellgrund anführten, haben das Rupertusblatt auch als Abonnenten nie gelesen und sind zu zwei Drittel NieGottesdienst-Besucher. Diese zwei Drittel von ihnen lehnen klare Gebote der Kirche und den Satz „Gott spricht durch die Kirche“ entschieden ab. Alle Befragten aus dieser Abbestellergruppe sind der Ansicht, auch ohne Gottesdienstbesuch könne man ein guter Christ sein. Durchwegs lehnen sie die Notwendigkeit der „Ehrfurcht“ vor der Kirche ab.

\section{Abbestellgrund: Beenden einer "Tradition“}

Nur jeder sechste der „Abonnenten aus Tradition“ unter den Abbestellern hat die Kirchenzeitung auch regelmäßig gelesen, zwei Drittel von ihnen sind deklarierte „NieLeser $^{\star}$. Diese Personen sind fast zur Hälfte Nie-Gottesdienstbesucher, zur anderen Hälfte besuchen sie gelegentlich den Gottesdienst. Keiner aus dieser Gruppe gab an, dem Sonntags-Gottesdienst regelmäßig beizuwohnen. Drei Viertel von ihnen sind als kirchenfern einstufbar.

\section{Abbestellgrund: Nur Verwandten „zuliebe" abonniert}

Diese Gruppe von Stornierern deckt sich (in ihren Merkmalen) zu einem guten Teil mit den "Traditionsbeendern“. Mehr als drei Viertel von ihnen haben das Rupertusblatt nie gelesen - sie sind in einer überwiegenden Mehrheit als kirchenfern einzuschätzen. Immerhin 70 Prozent aus dieser Gruppe führten an, nie den Sonntags-Gottesdienst zu besuchen.

\section{Abbestellgrund: Pfarrbrief genügt als Information}

Diese Gruppe von Stornierern weist ein nur geringes Informationsbedürfnis auf. Es handelt sich dabei vorwiegend um jene „Nicht-Leser“, die jedoch eine höhere Kirchlichkeit aufweisen.

\section{Abbestellgrund: Fehlen der Ortsnachrichten}

Jene Abbesteller, die das Fehlen von Ortsnachrichten beklagten, waren zur Hälfte regelmäßige Leser des Diözesanblattes. Sie sind jedoch zum überwiegenden Teil als eher kirchenfern einzuschätzen. Weniger als ein Viertel aus dieser Gruppe kann als eher kirchennah beurteilt werden.

\section{Summary}

A very large proportion of subscription cancellations is due to the natural cause of death and to a general tendancy away from the Church in society. Only in a few instances is dissatisfaction with the product the real, decisive reason for cancellation. As many as $45 \%$ of the subscribers who were asked how thoroughly they read the "Rupertusblatt ${ }^{\text { }}$ replied that they seldom or never read it. Just about a third of those who cancelled their subscription replied that they had read the "Rupertusblatt" regularly. Thus it can be assumed that, leaving aside those who died, almost half of those cancelling their subsciption had already „broken“ with their church paper. This group of respondents is also characterized by a general alienation from church and an increasing erosion of church allegiance. It is certainly a matter for reflection that those who cancel their subscription to a church magazine have no great sense of church allegiance in general, such as a whole series of religio-sociological investigations ascribe to the average Catholic. For it certainly had to be presumed that those who were subscribers at least up to recently belonged rather to the more church- 
orientated and religious category of Catholics. The most common and certainly most "welcome" reason given for cancelling subsciptions is "lack of time". About half of those interviewed gave this as one reason, about a quarter gave it as the main reason. For every tenth person cancelling the main reason given for doing so was subscription costs. This group is characterized by relatively high sense of church allegiance and religiosity. Of those who no longer subscribe, roughly one out of seven said that basically they had no interest in church or religion. About a fifth took out a subscription to the church newspaper "just out of tradition" or for social reasons (relatives, family etc.).

\section{Résumé}

Beaucoup de ristournes d'abonnements résultent d'une perte "naturelle " par décès ou d'une tendance générale de sécularisation dans la socièté. C'est très rare qu'un mécontentement concret du prodiut soit la raison vraiment déclenchante et décisive de la cessation de l'abonnement. A la question de l'intensité personnelle de la lecutre du „Rupertusblatt“, $45 \%$ ont répondu qu'en tant qu'abonnés, ils n'avaient lu le "Rupertusblatt“ que rarement ou méme jamais. Moins d'un tier des

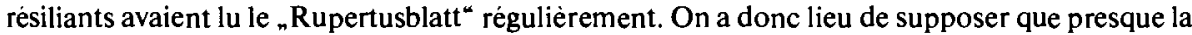
moitie des résiliants - les cas de décès n'y sont pas inclus - aient déjà "rompu“ avec leur journal de l'Eglise avant de faire cesser l'abonnement. Ce groupe des gens interrogés se fait aussi remarquer par une distance générale de l'Eglise. Le fait que ceux qui ont fait cesser leur abonnement ne sont guère mieux pensants que la moyenne des catholiques - selon toute une série d'analyse socioreligieuses - , certainement mérite réflexion. On aurait du penser que ceux qui comptaient parmi les abonnés il y a peu de temps, faisaient partie du segment plus ecclésiastique et religieux des ca-

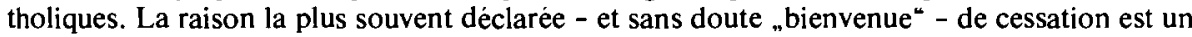
"manque de temps“. A peu près la moitie des gens interrogés allèguent ceci comme une raison, pour un quart c'est la raison principale. Pour un resiliant sur dix, les coùts de l'abonnement sont décisifs pour la cessation de l'abonnement. Ce groupe se fait remarquer par une religiosité comparativement grande. Un ancien abonné sur sept déclare qu'il se désintéresse par principe de l'Eglise et de la religion. A peu près un cinquième des gens interrogés n'avaient abonné le journal de l'Eglise que pour des raisons de "tradition" ou des raison sociales (parenté, famille etc.).

\section{Resumen}

Gran parte de las anulaciones de suscriptiones resulta de una terminación "natural“ a causa de muerte y de una tendencia general de alejamiento de la iglesia en la sociedad. Solamente en casos individuales el motivo definitivo para anular la suscripción consiste en un descontento con el producto. Interrogados por la intensidad de lectura personal de periodico "Rupertusblatt" respon-

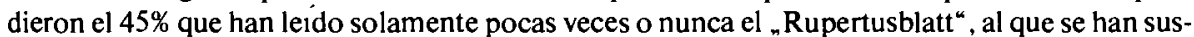
crito. Solo una tercera parte de las personas interrogadas que han anulado la suscripción solia leer con regularidad el "Rupertusblatt ${ }^{\star}$, según afirman ellos mismos. Asi que se puede concluir que casi la mitad de las personas que anulan la suscripción - excepto los casos de muerte - habian „roto" con su periódico ya antes de efectuar la anulación. Este grupo, al mismo tiempo, es caracterizado por un alejamiento general de la iglesia y por una erosión de su devoción. Seguramente debe notarse que la totalidad de las personas interrogadas que han anulado la suscripcion del periódico eclesiástico, no muestran mucho mas devoción, que lo que se sabe por medio de una serie de investigaciones socio-religiosas del católico medio. No obstante deberia tenerse en cuenta que los que todavia contaron entre los suscriptores hasta hace poco, figuran entre el segmento más eclesiástico y religioso de los católicos. El motivo para anulación mencionado con mayor frecuencia - y seguramente el motivo más „oportuno“ - es el de falta de tiempo. A proximadamente la mitad de los interrogados menciona este motivo como uno entre otros; para aproximadamente una cuarta parte de ellos es el motivo principal. Para cada diez en cien personas que han anulado la suscripción son los costos que los levan a terminar la suscripción. Este grupo es caracterizado por alta devoción y religiosidad, comparado con otros. A proximadamente siete en cien de los antiguos suscritores que han sido interrogados manifiestan su desinterés fundamental en la iglesia y la religión. Aproximadamente la quinta parte de los interrogados se ha suscrito al periódico de la igelsia "solamente por motivos de la tradición" o por razones sociales (familiares, etc.). 